\title{
Physician Payment Sunshine Act: Orthopedic Surgeons What You Should Know
}

\author{
Thomas Sullivan, President, Rockpointe, Abraham Gitterman, JD Rockpointe
}

$\mathrm{D}$ espite the overwhelming benefits that physician-industry collaboration and innovation have created over the last century, politicians, members of academia, and other public citizen groups have increasingly expressed their concern that physician-industry relationships may raise ethical issues, such as potential conflicts of interest. As a result of these concerns, Congress passed the Physician Payments Sunshine Act ("Sunshine Act"), section 6002 of the Affordable Care Act, in 2009.

The Sunshine Act, now called Open Payments, does not directly change or prohibit relationships between manufacturer's, physicians or teaching hospitals. Rather, the legislation and regulations require applicable pharmaceutical, biological and device manufacturers and group purchasing organizations (GPO), to report certain payments such entities make to physicians or teaching hospitals.

The definition of "physician" includes all licensed MDs, DOs, Dentists, Dental Surgeons, Podiatrists, Optometrists, or Chiropractors, regardless of whether they are currently practicing or whether they are enrolled with CMS. Non-U.S. physicians are excluded, but several countries have enacted similar legislation (e.g., France, U.K., Slovakia, Russia and the Netherlands). The Sunshine Act does not apply to pharmacists, physician's assistants, nurses, or nurse practitioners, though indirect payments meant paid to these individuals meant to pass through to a physician are reportable. The Sunshine Act also does not apply to medical residents, however, CMS recently clarified in its Frequently Asked Questions (FAQ) that payments to "fellows" are reportable.

Manufacturers must begin reporting their payments to physicians and teaching hospitals on August 1,2013. The first "payment reports" will collect data through December 31, 2013 (with the first report due March 31, 2014). Reporting is required annually thereafter. A manufacturer or GPO that fails to report or reports inaccurately may face annual fines up to $\$ 1.15$ million dollars. However, physicians face no fines or penalties under this law.

Once manufacturers collect, record and report the required information, the Center for Program Integrity at the Centers for Medicare \& Medicaid Services (CMS), which issued the final regulations and is responsible for the oversight and implementation of this program, will aggregate payments to all physicians and teaching hospitals and post them on a searchable, public website sometime after September 30, 2014.

Manufacturers and GPOs will have to report certain payments or transfers of value to physicians and teaching hospitals for categories including, but not limited to meals, travel, clinical research, honorarium, charitable contributions, consulting, speaking at promotional events, gifts, royalties or licenses and grants. For each of these categories, manufacturers and GPOs will also have to report the form of payment: 1) cash; 2) in-kind items or services; 3) stock, stock option or other ownership interest; and 4) dividend, profit or other return on investment.

Importantly, if a payment or transfer of value is related to marketing, education or research, including compensation for speaking, the manufacturer or GPO must also report the associated covered drug, device, biological or medical supply associated with that transaction.

Brennan, Manna \& Diamond

Attorneys \& Counselors at Law, www.bmdllc.com

Akron, Ohio 


\section{Impact on Orthopedic Surgeons}

First, as a reminder, all physicians should locate the National Plan and Provider Enumeration System (NPPES) website, and ensure that the following information is up-to-date and accurate: 1) name (first, last and middle); 2) mailing address; 3) primary practice address (and secondary if applicable); 4) specialty, including any subspecialty (particularly those in general practice, e.g., internal medicine); 5) National Provider Identifier (NPI) number; and 6) state license number(s).

Second, physicians should register with the CMS Open Payments website to receive updates and notifications. More importantly, registration will allow CMS to notify doctors when they have aggregated all payments in a reporting year and such payments are ready for review by the physician. Specifically, once physicians receive notice, they will have 45 days to review them. If the physician believes there is an inaccuracy, they may notify CMS of the problem. The agency will notify the manufacturer. However, CMS will not be responsible for resolving the dispute. An additional 15 days will be provided to resolve the dispute. If it is unresolved after 60 days, CMS will publish the payment as the manufacturer has reported it, indicating that it is "disputed."

Next, orthopedic surgeons must be aware of certain reporting requirements that are unique to this specialty. Orthopedic surgeons likely use a combination of both drugs, devices, biological, and medical supplies - all of which reporting is required.

With respect to devices, there are a number of categories of payments or transfers of values that a medical device manufacturer may give to a surgeon that do not require reporting or tracking. For example, CMS excluded from reporting short-term loans of a covered device between 1-90 days. In the final rule, CMS expanded this exclusion to include covered devices "under development" including "a supply of disposable or single use devices (including medical supplies) intended to last for no more than 90 days." [1] In addition, CMS clarified that once a short-term loan exceeds the 90-day exclusion period, "regardless of whether the days were consecutive," the manufacturer must begin reporting from the 91st day. [2] Further, if a covered recipient purchases the device within the 90 days, manufacturers do not need to report the loan. Thus, surgeons will need to be aware of the timing of any loans and purchasing

In addition, CMS clarified that certain medical devices may be excluded from reporting as "product samples" while others may fall under the "shortterm loan" exclusion. Specifically, CMS reasoned that "single use or disposable devices, demonstration devices or evaluation equipment" are excluded from reporting as samples as long as they "are intended for use by patients"; otherwise, such items "may be excluded from reporting ... [as] short term loans." [3] Thus, surgeons should ask whether a manufacturer classifies the product as a sample or short term loan and have staff record such information so a surgeon is not later surprised when a payment appears on the Open Payment website.

Surgeons will also need to pay close attention to contractual warranties offered by manufacturers or GPOs. CMS finalized that contractual warranty exclusions may extend to items and services provided "outside the expiration period" "as long as the contractual warranty specified in the terms prior to expiration." [4] In addition, CMS finalized that "items or services provided under a contractual service or maintenance agreement" are also subject to the contractual warranty exclusion because they are "so similar to warranty agreements." [5]

There are also unique challenges for reporting research and consulting arrangements. Specifically, surgeons must understand that only payments included in a written research agreement or protocol are eligible for delayed publication as "research payments." Because of the frequent interactions medical device manufacturers must have with surgeons to develop medical technologies, manufacturers will not always be able to capture in a written agreement or research protocol each interaction necessary for the research and development of a medical device or technology - before, during and after FDA clearance.

As a result, meals, travel, education, consulting, honoraria or training associated with the $R \& D$ of a medical device or technology will likely become separately reportable in their respective categories, even though they are associated with the research. Accordingly, surgeons must be aware of these special rules to understand that some payments will not be delayed in publication even though they are related to research.

Similarly, because CMS finalized that clinical investigations of new applications of existing products are not eligible for delayed publication, surgeons will need to ask manufacturers about when certain products are not eligible for delayed payment, and 
thus, payments related to the 'non-delayable' research (e.g., travel, meals, etc.) will also have to be reported separately. Surgeons conducting clinical research and trials should also work with manufacturers to determine who is responsible for reporting the costs of medical devices and supplies in the "total amount of research payment" that is eligible for publication delay.

Another area is ownership interests, if an orthopedic surgeon is receiving royalty, stock or options in return for work in developing innovative medical products those payments will be reported. In addition if a surgeon owns stock or options in a medical products or GPO company not being traded in a public exchange the value of that stock will be reported each calendar year. This includes private stock held by immediate family members including spouse, children, grandparents, grandchildren, siblings and their spouses if their relationship is known to the company.

\section{Surgeons and Patients}

Finally, surgeons should begin checking all of their current or near future contracts with GPOs and manufacturers to ensure that reporting requirements and obligations are met. Importantly, surgeons that are members of guidelines committees or professional medical associations, or who work working at academic institutions will need to ensure that any relationships with industry do not exceed what are permitted by the policies governing these institutions or groups.
In addition, doctors should update any and all conflict of interest disclosure forms or slides they may use in teaching and educational activities and with their institution, as well as any authorship or journal publications. Doctors will also need to pay close attention to past, present, and future filings with the IRS and any potential tax implications payment reporting may have, particularly with respect to royalties and licenses.

Lastly, it will be critical to ensure that doctors inform patients about any relevant relationships they may have with manufacturers prior to beginning a new course of treatment or recommending surgery. Some states have strong consumer protection laws and physicians may be held liable for failing to inform patients regarding certain relationships, which may raise lack of informed consent issues.

Accordingly, surgeons must be ready to discuss such payments with patients in a neutral way to ensure that patients understand the nature of the relationship and why these interactions are essential to lifesaving medical breakthroughs, the development of new medicines, and improvements in the care we all receive.

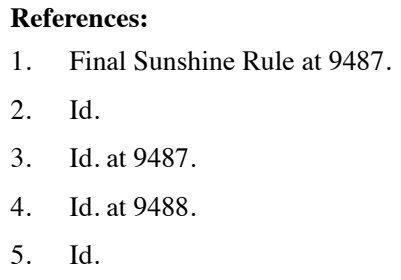

